Kardiologe 2021 · 15:121-126

https://doi.org/10.1007/s12181-021-00446-z

Angenommen: 18. Januar 2021

Online publiziert: 24. Februar 2021

(c) Deutsche Gesellschaft für Kardiologie -

Herz- und Kreislaufforschung e.V. Published by

Springer Medizin Verlag $\mathrm{GmbH}$, ein Teil von

Springer Nature - all rights reserved 2021

\author{
Christoph Birner ${ }^{1} \cdot$ Ulrich Laufs $^{2} \cdot$ Norbert Frey $^{3}$ \\ 'Klinik für Innere Medizin I, Klinikum St. Marien Amberg, Amberg, Deutschland \\ ${ }^{2}$ Klinik und Poliklinik für Kardiologie, Universitätsklinikum Leipzig, Leipzig, Deutschland \\ ${ }^{3}$ Klinik für Innere Medizin III: Kardiologie, Angiologie und Pneumologie, Universitätsklinikum Heidelberg, \\ Heidelberg, Deutschland
}

\title{
Neue Therapiekonzepte für die Herzinsuffizienz
}

\section{Studien-Highlights 2020}

Ohne Frage stand das Jahr 2020 unter dem Eindruck der COVID-19-Pandemie, die in besonderem Maße auch herzinsuffiziente Patienten bedroht. So zeigte sich bereits in einer frühen Studie mit 799 Patienten, dass die Herzinsuffizienz mit einer Inzidenz von $24 \%$ bei allen COVID-19-Erkrankten und von $49 \%$ bei denen mit letalem Verlauf zu den häufigsten Komplikationen dieser pandemischen Erkrankung gehört [6]. Die genauen Mechanismen der dokumentierten myokardialen Beteiligung sind noch nicht ganz klar. Zusätzlich verstärkt die COVID-19-Infektion die ohnehin hohe Krankheitslast der Patienten mit Herzinsuffizienz. Wie groß diese Last gerade bei der Herzinsuffizienz ist, konnte eine beim virtuellen Kongress der HFA (Heart Failure Association) vorgestellte Studie zeigen [7]: 2017 war die Herzinsuffizienz nicht nur der häufigste Grund für eine Klinikeinweisung in Deutschland und dabei auch die mit Abstand häufigste Todesursache bei den dann auch im Krankenhaus verstorbenen Patienten -, sondern die Hospitalisierungshäufigkeit nahm zwischen 2000 und 2017 sogar um beachtliche $94 \% \mathrm{zu}$. Angesichts dieser Tatsachen wird erkennbar, wie dringend erforderlich zusätzliche, innovative Therapieansätze für die Herzinsuffizienz sind.

Einige davon - medikamentöse wie interventionelle - wurden 2020 in Studien untersucht, deren Ergebnisse überwiegend hoffnungsvoll stimmen (s. auch - Tab. 1):

\section{Medikamentöse Therapie}

\section{SGLT2-Inhibitoren}

\section{Was bisher bekannt war}

Durch eine selektive Hemmung des „sodium dependent glucose cotransporter 2" (SGLT2) im proximalen Nierentubulus reduzieren diese Medikamente die renale Glukosereabsorption und erhöhen damit die Glukoseausscheidung. Insofern waren die Gliflozine zunächst als Antidiabetika entwickelt worden. In der nachfolgend an Typ2-Diabetikern durchgeführten EMPAREG Outcome-Studie zeigte sich dann überraschenderweise eine über die bloße Blutzuckersenkung hinausgehende, signifikante Reduktion von kardiovaskulärer Mortalität, Gesamtmortalität und auch Hospitalisierung wegen einer Herzinsuffizienz [26]. Gerade letzteres Resultat führte in der DAPA HFStudie zu einer Evaluierung der kardiovaskulären Endpunkte in einem dezidierten HFrEF(Herzinsuffizienz mit eingeschränkter Ejektionsfraktion)-Kollektiv. Interessanterweise konnten auch hier diese positiven Effekte nachgewiesen werden - und zwar unabhängig davon, ob die Patienten an einem Diabetes mellitus Typ 2 litten oder nicht [10].

\section{Was 2020 hinzukam}

Ähnlich wie die DAPA HF-Studie, untersuchte auch die 2020 veröffentlichte EMPEROR-Reduced-Studie SGLT2Inhibitoren an einem HFrEF-Kollek- tiv. Ein Unterschied zwischen beiden Studien war, dass EMPEROR-Reduced Patienten mit einer etwas weiter fortgeschrittenen systolischen Dysfunktion einschloss. Auch hier konnte der kombinierte Endpunkt (kardiovaskulärer Tod oder Hospitalisierung wegen einer sich verschlechternden Herzinsuffizienz) signifikant gesenkt werden, allerdings war dieses Ergebnis vorwiegend durch die reduzierte Hospitalisierung bedingt. Darüber hinaus verbesserte Empagliflozin signifikant renale Endpunkte. Wichtig war nun, dass sich diese Vorteile erneut unabhängig vom Vorliegen eines Diabetes mellitus zeigten - und dass sie darüber hinaus selbst bei den mit einem ARNI (Angiotensin-RezeptorNeprilysin-Inhibitor) und somit optimal behandelten Patienten nachweisbar waren [11].

Eine spannende Frage war ferner, inwieweit die offensichtlich bei HFrEF so wirksamen SGLT2-Inhibitoren auch bei HFpEF (Herzinsuffizienz mit erhaltener Ejektionsfraktion) oder einer akut dekompensierten Herzinsuffizienz effektiv sein könnten, also bei Entitäten, für die es weit weniger etablierte medikamentöse Therapiekonzepte gibt.

Hier setzte die SOLOIST-WHF-Studie an, die Sotagliflozin vs. Placebo bei Typ-2-Diabetikern nach akuter kardialer Dekompensation untersuchte [5]. Auch wenn diese Studie an methodischen Mängeln litt, war doch erkennbar, dass SGLT2-Inhibitoren auch bei kürzlich dekompensierten Patienten kardiovaskuläre Endpunkte reduzieren 


\section{Schwerpunkt}

Tab. 1 Studien-Highlights 2020 zur Therapie der Herzinsuffizienz

\begin{tabular}{|c|c|c|c|c|}
\hline & & AHF/ADHF & CHF & \\
\hline & & & HFrEF & HFpEF \\
\hline $\begin{array}{l}\text { Medika- } \\
\text { mentöse } \\
\text { Therapie }\end{array}$ & $\begin{array}{l}\text { SGLT2-In- } \\
\text { hibitoren }\end{array}$ & $\begin{array}{l}\text { SOLOIST-WHF } \\
1222 \text { Typ-2-Diabetiker mit dekom- } \\
\text { pensierter Herzinsuffizienz; Sotag- } \\
\text { liflozin vs. Placebo: CV-Tod oder } \\
\text { WHF-Hospitalisierung/-Vorstellun- } \\
\text { gen } \downarrow \text { auch bei HFpEF }\end{array}$ & $\begin{array}{l}\text { EMPEROR-Reduced } \\
3730 \text { HFrEF-Patienten; OMT + } 10 \text { mg Em- } \\
\text { pagliflozin oder Placebo: CV-Tod oder } \\
\text { WHF-Hospitalisierung } \downarrow \text { bei Diabetikern } \\
\text { und Nicht-Diabetikern; Verbesserung } \\
\text { renaler Endpunkte }\end{array}$ & $\begin{array}{l}\text { SOLOIST-WHF } \\
\text { (s. AHF/ADHF) }\end{array}$ \\
\hline & ARNI & - & $\begin{array}{l}\text { PIONEER HF-Subgruppenanalyse } \\
\text { Sacubitril/Valsartan vs. Enalapril bei } \\
881 \text { HFrEF-Patienten mit stabilisierter } \\
\text { akut dekompensierter Herzinsuffizienz: } \\
\text { positive Effekte auch bei De-novo-HFrEF } \\
\text { und ohne RAS-I-Vorbehandlung }\end{array}$ & $\begin{array}{l}\text { PARALLAX } \\
2572 \text { HFpEF-Patienten; Sacubitril/ } \\
\text { Valsartan vs. RAS-I (Enalapril, Valsar- } \\
\text { tan, Placebo): NT-proBNP } \downarrow \text {, kein Effekt } \\
\text { auf 6-min-Gehtest. Post-hoc-Analyse: } \\
\text { WHF-Hospitalisierung } \downarrow\end{array}$ \\
\hline & $\begin{array}{l}\text { sGC- } \\
\text { Stimu- } \\
\text { latoren }\end{array}$ & - & $\begin{array}{l}\text { VICTORIA } \\
\text { Vericiguat vs. Placebo bei } 5050 \text { Patienten } \\
\text { mit LVEF }<45 \% \text { und WHF: CV-Tod oder } \\
\text { WHF-Hospitalisierung } \downarrow\end{array}$ & $\begin{array}{l}\text { VITALITY-HFpEF } \\
\text { Vericiguat vs. Placebo bei } 789 \mathrm{HFpEF-} \\
\text { Patienten mit Zustand nach kardialer } \\
\text { Dekompensation: keine Veränderung der } \\
\text { funktionellen Kapazität }\end{array}$ \\
\hline & & & - & $\begin{array}{l}\text { CAPACITY-HFpEF } \\
\text { Praliciguat vs. Placebo bei } 196 \mathrm{HFpEF-Pa-} \\
\text { tienten: keine Verbesserung der } \mathrm{VO}_{2} \text { max } \\
\text { in der Ergospirometrie }\end{array}$ \\
\hline & Myosinakt & - & $\begin{array}{l}\text { GALACTIC-HF } \\
\text { Omecamtiv mecarbil vs. Placebo bei } \\
8256 \text { HFrEF-Patienten: CV-Tod oder WHF } \downarrow \\
8 \%\end{array}$ & - \\
\hline $\begin{array}{l}\text { Inter- } \\
\text { ventionelle } \\
\text { Therapie }\end{array}$ & $\begin{array}{l}\text { Klappen- } \\
\text { therapie }\end{array}$ & - & $\begin{array}{l}\text { International MitraBridge Registry } \\
119 \text { HFrEF-Patienten mit sekundärer MI } \\
\text { und Indikation zur HTX: nach MitraClip } \\
\text { (Abbott, Abbott Park, IL, USA) bei } 24 \% \\
\text { keine HTX mehr indiziert, bei } 16 \% \text { lis- } \\
\text { tungsfähiger Zustand erreicht }\end{array}$ & - \\
\hline & BAT & - & $\begin{array}{l}\text { BeAT HF } \\
\text { BAT + OMT vs. OMT bei } 245 \text { HFrEF-Patien- } \\
\text { ten: QOL } \uparrow, 6 M W T \uparrow, \text { NT-proBNP } \downarrow\end{array}$ & - \\
\hline & ECMO & $\begin{array}{l}\text { ARREST } \\
\text { VA-ECMO vs. ACLS bei } 30 \text { OHCA- } \\
\text { Patienten mit VF ohne ROSC nach } \\
3 \text { Defibrillationen: Überleben bis } \\
\text { Krankenhausentlassung: } 43 \% \text { vs. } \\
7 \%\end{array}$ & - & - \\
\hline $\begin{array}{l}\text { ACLS erweite } \\
\text { ARNI Angiote } \\
\text { geschränkte } \\
\text { trahospitaler } \\
\text { giotensin-Sys } \\
\text { „Sodium dep } \\
\text { stoffaufnahm }\end{array}$ & $\begin{array}{l}\text { erzstillstand } \\
\text { m-Inhibitor, } \\
\text { dent glucose }\end{array}$ & $\begin{array}{l}\text { en der Wiederbelebung (,advanced car } \\
\text {-Neprilysin-Inhibitor, BAT Barorezeptor } \\
\text { : HFrEF), CV kardiovaskulär, HTX Herztra } \\
\text { („Out of hospital cardiac arrest"), OMT o } \\
\text { ROSC Wiederetablierung eines sponta } \\
\text { e cotransporter 2“, VA-ECMO venoarter }\end{array}$ & $\begin{array}{l}\text { ac life support"), } A H F \text { akute Herzinsuffizienz, } A \\
\text { ivierungstherapie, CHF chronische Herzinsuffi } \\
\text { plantation, } L V E F \text { linksventrikuläre Ejektionsfrak } \\
\text { imale medikamentöse Therapie, } Q O L \text { Lebensa } \\
\text { n Kreislaufs („return of spontaneous circulation } \\
\text { le extrakorporale Membranoxygenierung, VF }\end{array}$ & $\begin{array}{l}\text { DHF akut dekompensierte Herzinsuffizienz, } \\
\text { zienz (erhaltene Pumpfunktion: HFpEF, ein- } \\
\text { tion, MI Mitralklappeninsuffizienz, OHCA ex- } \\
\text { qualität („quality of life“), RAS-I Renin-An- } \\
\text { "), sGC lösliche Guanylatcyclase, SGLT2 } \\
\text { Kammerflimmern, } \mathrm{VO}_{2} \text { max maximale Sauer- } \\
\text { Gehtest }\end{array}$ \\
\hline
\end{tabular}

können. Interessanterweise ergab sich diese Wirksamkeit unabhängig von der LVEF (linksventrikuläre Ejektionsfraktion), war also auch bei den ca. $20 \%$ HFpEF-Patienten nachweisbar.

Gerade diese Feststellung ist angesichts der bekanntermaßen unzureichenden HFpEF-Therapiemöglichkeiten außerordentlich spannend, allerdings war hierfür die SOLOIST-WHF-Studie nicht gepowert. Diesbezüglich müssen also die Ergebnisse u.a. der noch laufenden DELIVER- und EMPERORPreserved-Studie abgewartet werden.

\section{Welches klinische Fazit ergibt sich daraus?}

SGLT2-Inhibitoren werden künftig einen wichtigen Stellenwert bei der HFrEF-, möglicherweise sogar bei der HFpEF-
Therapie einnehmen (• Abb. 1). Konsequenterweise empfiehlt daher die Heart Failure Association der Europäischen Gesellschaft für Kardiologie (ESC) bereits, SGLT2-Inhibitoren bei ansonsten optimal behandelten, weiterhin symptomatischen HFrEF-Patienten einzusetzen und zwar unabhängig davon, ob zusätzlich ein Diabetes mellitus Typ 2 vorliegt oder nicht [15]. 
ARNI (Angiotensin-RezeptorNeprilysin-Inhibitor)

\section{Was bisher bekannt war}

Während Sacubitril/Valsartan bei HFrEF-Patienten zu einer signifikanten Reduktion von Morbidität und Mortalität führt [9], waren die Ergebnisse für $\mathrm{HFpEF}$ bisher weniger überzeugend. Immerhin ließen sich in der 2019 veröffentlichen PARAGON HF-Studie vornehmlich in 2 Subgruppen (Frauen und Patienten mit einer LVEF zwischen 45 und $57 \%$ ) positive Effekte auf den kombinierten primären Endpunkt aus kardiovaskulärem Tod und Herzinsuffizienzhospitalisierung erkennen [17].

\section{Was 2020 hinzukam}

Leider setzten sich die ernüchternden Ergebnisse für HFpEF-Patienten auch in der 2020 vorgestellten PARALLAXStudie fort: Hier ergab sich zwar eine signifikante Reduktion des NTproBNP-Spiegels, allerdings blieb der zweite, koprimäre Endpunkt (Distanz im 6-min-Gehtest) unbeeinflusst. Immerhin ergab eine Post-hoc-Analyse ein signifikant um $50 \%$ reduziertes Risiko für eine Herzinsuffizienz-bedingte Hospitalisierung [12]. Demgegenüber ließ sich in einer ebenfalls 2020 veröffentlichten Subgruppenanalyse der PIONEER-HF-Studie für HFrEF zeigen, dass selbst diejenigen Patienten, die mit einer akut dekompensierten, erstmals diagnostizierten Herzinsuffizienz ohne ACE(Angiotensin-Converting-Enzym)Hemmer- bzw. Sartan-Vorbehandlung noch bei PARADIGM HF ausgeschlossen waren, signifikant von Sacubitril/ Valsartan profitieren [1]. Die TRANSITION-Studie bestätigte zudem die Sicherheit einer primären Initiierung der Therapie im Krankenhaus auch bei der Erstdiagnose einer Herzinsuffizienz $[16,22]$.

\section{Welches klinische Fazit ergibt sich daraus?}

Während sich somit auf der einen Seite die große Bedeutung von Sacubitril/ Valsartan für die HFrEF-Behandlung - auch als First-line-Therapie - bestätigt (• Abb. 1), überzeugen auch die neueren Daten für HFpEF nicht. Viel Optimie- rungspotenzial gibt es in Deutschland noch bezüglich des Einsatzes der vollen Dosierungen [23].

\section{sGC-Stimulatoren}

\section{Was bisher bekannt war}

Seit Längerem schon weiß man, dass der molekulare cGMP-PKG(cyclische Guanosinmonophosphat-abhängige Protein Kinase G)-Weg potenziell kardioprotektive Eigenschaften besitzt. Der hierfür zentrale „second messenger“ cGMPkann dabei entweder durch die lösliche (sGC) oder aber durch die membranständige partikuläre Guanylatcyclase (pGC) gebildet werden. Während Letztere dem natriuretischen Peptidrezeptor entspricht und somit durch natriuretische Peptide aktiviert werden kann, dient Stickstoffmonoxid als natürlicher Aktivator der sGC. In den letzten Jahren gelang es nun, Substanzen zu entwickeln, die die enzymatische Aktivität der sGC auf pharmakologischem Weg erhöhen können (sog. sGC-Stimulatoren). Trotz der postulierten, großen Bedeutung des cGMP-PKGWeges in der Herzinsuffizienz blieben bisherige klinische Studien hinter den Erwartungen zurück.

\section{Was 2020 hinzukam}

Vor diesem Hintergrund gab die 2020 veröffentlichte VICTORIA-Studie wieder Anlass zur Hoffnung: Hier nämlich wurde der orale sGC-Stimulator Vericiguat an Patienten mit einer LVEF < $45 \%$ und kürzlich dekompensierter Herzinsuffizienz (HF) untersucht und eine $10 \%$ ige Reduktion des kombinierten Endpunkts aus kardiovaskulärem Tod und Herzinsuffizienz-bedingter Hospitalisierung für Vericiguat nachgewiesen. Der Effekt bei den Patienten mit schwerer HF zeigte sich dabei zusätzlich zu einer guten, etablierten leitliniengerechten Therapie. Auch wenn dieses Ergebnis vornehmlich durch eine Abnahme von Hospitalisierungen zustande kam, zeigte sich damit dennoch, dass das pathophysiologische Konzept einer sGC-vermittelten cGMP-Erhöhung bei HFrEF vorteilhaft sein kann [3]. Leider ließen sich diese hoffnungsvollen Signale bei den beiden ebenfalls 2020 veröffentlichten HFpEF-Studien VITALITY-HFpEF
Kardiologe 2021 · 15:121-126 https://doi.org/10.1007/s12181-021-00446-z (c) Deutsche Gesellschaft für Kardiologie Herz- und Kreislaufforschung e.V. Published by Springer Medizin Verlag $\mathrm{GmbH}$, ein Teil von Springer Nature - all rights reserved 2021

\section{Birner · U. Laufs · N. Frey}

Neue Therapiekonzepte für die Herzinsuffizienz. StudienHighlights 2020

\section{Zusammenfassung}

Die Versorgung von Patienten mit Herzinsuffizienz ist in Pandemiezeiten eine besondere Herausforderung, da die Vulnerabilität kardiovaskulär erkrankter Patienten für die COVID-19Infektion zu einer weiteren Erhöhung der Herzinsuffizienzmorbidität und -mortalität führen könnte. Gleichzeitig weisen viele 2020 publizierte Studien auf günstige Effekte innovativer Therapieprinzipien hin. Bei der Therapie der systolischen Herzinsuffizienz (HFrEF [heart failure with reduced ejection fraction]) rücken SGLT2 (,sodium dependent glucose cotransporter 2")-Inhibitoren und Sacubitril/Valsartan in die erste Reihe.

\section{Schlüsselwörter}

Medikamentöse Therapie · Interventionelle Therapie · Akute Herzinsuffizienz .

Chronische Herzinsuffizienz - Kardiogener Schock

Novel treatment concepts for heart failure. Study highlights 2020

\section{Abstract}

It is to be feared that the great vulnerability of patients with cardiovascular diseases to the coronavirus disease 2019 (COVID19) pandemic will further heighten the already enormous burden of morbidity and mortality of heart failure. Many of the clinical trials published in 2020 show the favorable effects of innovative treatment principles. For the treatment of systolic heart insufficiency (heart failure with reduced ejection fraction, HFrEF) sodium-glucose cotransporter 2 (SGLT2) inhibitors and sacubitril/valsartan are prioritized.

Keywords

Pharmacotherapy - Interventional therapy Acute heart failure - Chronic heart failure . Cardiogenic shock 


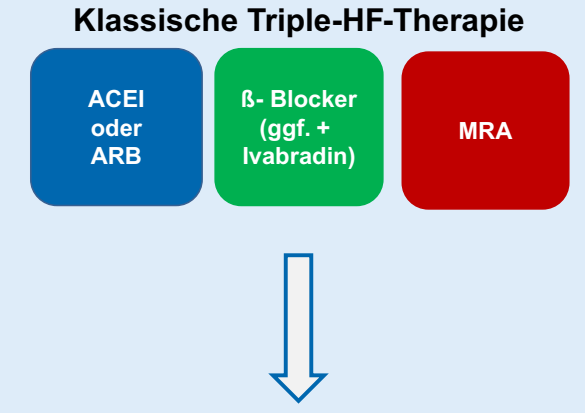

2020: 4-Säulen-Therapie zur Lebensverlängerung bei Herzinsuffizienz
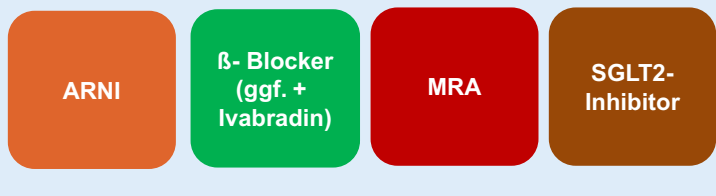

Abb. 1 A Entwicklung der lebensverlängernden Therapien für die systolische Herzinsuffizienz (HF). ACE-I ACE-Hemmer, ARB Angiontensin-Rezeptorblocker, MRA Mineralokortikoidrezeptor-Antagonist, $A R N I$ Angiotensin-Rezeptor-Neprilysin-Inhibitor, SGLT2 "sodium dependent glucose cotransporter 2"

und CAPACITY-HFPEF nicht finden: Während Erstere die Auswirkungen von Vericiguat auf die funktionelle Kapazität untersuchte [2], analysierte Letztere den Effekt des sGC-Stimulators Praliciguat auf den $\mathrm{VO}_{2} \max$ (maximale Sauerstoffaufnahme)-Wert in der Spiroergometrie [21]. Diesen beiden Studien war gemeinsam, dass die Endpunkte jeweils verfehlt wurden.

\section{Welches klinische Fazit ergibt sich daraus?}

Insofern zeigt die 2020 hinzugekommene Evidenz, dass sich bei HFrEF die pharmakologische Beeinflussung des cGMPPKG-Weges in klinisch relevante Endpunkte übersetzen lässt. Dies gibt zumindest Anlass zur Hoffnung, dass mittelfristig sGC-Stimulatoren Einzug in das medikamentöse Armamentarium finden.

\section{Myosinaktivatoren}

\section{Was bisher bekannt war}

Der Myosinaktivator Omecamtiv mecarbil bindet im Sarkomer an Myosin und stabilisiert damit die Myosinköpfchen in einerStellung, in der sie mit Aktin in Kontakt treten und einen Kraftschlag auslösen können. Präklinische Studien konnten zeigen, dass Omecamtiv mecarbil dadurch die kardiale Performance verbes- sert, ohne den myokardialen Sauerstoffbedarf zu erhöhen [20].

\section{Was 2020 hinzukam}

Die 2020 veröffentlichte GALACTIC-HFStudie evaluierte nun, inwieweit dieses pathophysiologische Konzept in der Lage ist, klinische Endpunkte in einem HFrEFKollektiv zu beeinflussen: Bei über 8000 medikamentös adäquat vorbehandelten Patienten mit einer LVEF $\leq 35 \%$ wurde hier Omecamtiv mecarbil mit einer Placebogabe verglichen, wobei sich in der Verumgruppe eine signifikante, $8 \%$ ige Reduktion des kombinierten primären Endpunkts (CV[kardiovaskulärer]-Tod oder Verschlechterung der Herzinsuffizienz) ergab. Dieses Ergebnis wurde vorwiegend durch die Beeinflussung des Endpunktereignisses „Verschlechterung der Herzinsuffizienz" erreicht, auch beschränkten sich die gezeigten positiven Effekte in einer präspezifizierten Subgruppenanalyse auf Patienten mit einer LVEF unter dem Median, d.h. $\leq 28 \%$ [19].

\section{Welches klinische Fazit ergibt sich daraus?}

Die Effekte der GALACTIC-HF-Studie waren positiv, jedoch quantitativ eher moderat. Daher ist aktuell unklar, ob für die Substanz eine Zulassung in Deutschland beantragt wird.

\section{Interventionelle Therapie}

\section{Interventionelle Klappentherapie}

\section{Was bisher bekannt war}

Leitliniengerecht kann bei HFrEF-Patienten mit sekundärer Mitralklappeninsuffizienz eine kathetergestützte Edgeto-edge-Mitralklappenreparatur („MitraClip") erwogen werden, wenn sich keine Revaskularisationsmöglichkeit ergibt und darüber hinaus das Operationsrisiko zumindest nicht gering ist [4]. $\mathrm{Zu}$ bedenken ist allerdings, dass bei dieser mit „IIbC“ eher geringen Empfehlungsstärke die 2018 veröffentlichte COAPTStudie noch nicht berücksichtigt werden konnte, bei der sich eine signifikante Reduktion von Herzinsuffizienz-bedingter Hospitalisierung und Gesamtmortalität ergab [18].

\section{Was 2020 hinzukam}

Dass die MitraClip-Therapie selbst bei terminal herzinsuffizienten Patienten vorteilhaft sein könnte, lässt nun der 2020 publizierte Bericht des International MitraBridge Registry erahnen [8]: Hier wurden Patienten mit Indikation zur Herztransplantation (HTX) untersucht, die gleichzeitig unter einer relevanten sekundären Mitralklappeninsuffizienz litten. Auch wenn diese kleine und ohne Kontrollgruppe durchgeführte Studie allenfalls eine Hypothesengenerierung zuließ, war doch bemerkenswert, in welchem Ausmaß sich die Patienten nach MitraClip-Implantation stabilisierten: In ca. $16 \%$ konnte nach der Intervention überhaupt erst ein listungsfähiger $\mathrm{Zu}$ stand für eine HTX erreicht werden, und bei $24 \%$ gab es im Verlauf dann keine Indikation für eine HTX mehr.

\section{Welches klinische Fazit ergibt sich daraus?}

Sofern sich diese Ergebnisse in weiteren Studien erhärten lassen, würde dies die Bedeutung der MitraClip-Implantation als Methode der interventionellen Herzinsuffizienztherapie weiter untermauern. 


\section{Barorezeptoraktivierungstherapie (BAT)}

\section{Was bisher bekannt war}

Aktuell wird eine kardiale Resynchronisationstherapie (CRT) bei symptomatischen HFrEF-Patienten vorwiegend dann empfohlen, wenn ein Linksschenkelblock mit einer QRS-Dauer $\geq 150 \mathrm{~ms}$ (Klasse-IA-Empfehlung) bzw. 130-149 ms (Klasse-IB-Empfehlung) vorliegt [13]. Berücksichtigt man dabei allerdings die Tatsache, dass nur ca. $20 \%$ der herzinsuffizienten Patienten tatsächlich eine QRS-Dauer $\geq 120 \mathrm{~ms}$ aufweisen [13], wird erkennbar, dass für einen Großteil der HFrEF-Patienten eine kardiale Resynchronisationstherapie nicht infrage kommt. Insofern besteht ein großer Bedarf, auch diesen medikamentös häufig austherapierten Patienten eine zusätzliche Therapieoption anbieten zu können. Eine Möglichkeit wäre die gezielte Modulation des bei Herzinsuffizienz bekanntermaßen aktivierten autonomen Nervensystems.

\section{Was 2020 hinzukam}

Eine dieser Modulationstechniken ist die Barorezeptoraktivierungstherapie (BAT). Hierzu wurde 2020 die BeAT HFStudie veröffentlicht, in der 245 HFrEFPatienten entweder nur mit einer leitliniengerechten HFrEF-Therapie oder aber zusätzlich mit BAT behandelt wurden [25]. Bei letzterer Gruppe erfolgte die operative Platzierung einer Elektrode auf dem Karotissinus, die dann mit einem subkutan implantierten Impulsgenerator verbunden und stimuliert wurde. Unter der Einschränkung, dass diese Studie durchaus einige methodische Fallstricke hatte, ließ sich nachweisen, dass BAT in der untersuchten Kohorte zu einer signifikanten Verbesserung von Lebensqualität und 6-min-Gehtest sowie zu einer signifikanten Reduktion des NT-proBNP-Spiegels führte. Die Effekte bezüglich "harter" Endpunkte sind allerdings unbekannt.

\section{Welches klinische Fazit ergibt sich daraus?}

Diese Ergebnisse stellen einen Ausgangspunkt dar, diese Methode in einer Endpunktstudie für den Einsatz bei Patienten mit ausgereizter medikamentöser Therapie zu prüfen, für die eine CRT-Implantation nicht infrage kommt.

\section{ECMO-Therapie des kardiogenen Schocks bzw. nach Reanimation}

\section{Was bisher bekannt war}

Patienten mit extrahospitalem Herzstillstand (OHCA) haben bekanntermaßen eine außerordentlich schlechte Prognose selbst nach Initiierung der leitliniengerechten Reanimationsmaßnahmen („advanced cardiac life support" [ACLS]). Eine Möglichkeit zur Verbesserung der Überlebenschancen könnte die frühzeitige Implantation einer VA(venoarterielle)-ECMO (extrakorporale Membranoxygenierung) sein.

\section{Was 2020 hinzukam}

Dies zumindest lässt die 2020 veröffentlichte ARREST-Studie vermuten, in die 30 Patienten mit extrahospitalem Herzstillstand eingeschlossen wurden, bei denen das initial bestehende Kammerflimmern (bzw. die pulslose ventrikuläre Tachykardie) selbst nach 3 Defibrillationen nicht zu terminieren war [24]. Während die eine Gruppe nur mit den leitliniengerechten, erweiterten Maßnahmen der Wiederbelebung (ACLS) behandelt wurde, erhielt die andere Gruppe zeitnah eine VA-ECMO. Das bemerkenswerte Ergebnis, das auch zu einer vorzeitigen Beendigung der Studie geführt hatte, war nun, dass der primäre Endpunkt (Überleben bei Krankenhausentlassung) in der ECMO-Gruppe bei $43 \%$, in der ACLS-Gruppe nur bei $7 \%$ erreicht wurde. Und nach 6 Monaten waren von diesen ECMO-Patienten - bei guter Lebensqualität - noch alle am Leben, wohingegen keiner der ACLS-Patienten diesen Zeitpunkt erlebte. In einer weiteren Kohortenstudie von 686 Patienten zum Einsatz der VA-ECMO beim kardiogenen Schock zeigte sich, dass die pathophysiologisch sinnvoll erscheinende zusätzliche Entlastung des linken Ventrikels durch ein Impella-System (Abiomed, Danvers, MA, USA) die Mortalität weiter senkt [14].

\section{Welches klinische Fazit ergibt sich} daraus?

Insofern könnte die frühzeitige Implantation einer VA-ECMO - ggf. in Kombination mit einer Impella-Schraubenpumpe - möglicherweise $\mathrm{zu}$ einer Outcome-Verbesserung von OHCAPatienten beitragen. $\mathrm{Zu}$ beachten ist hier allerdings, dass die ARREST-Studie an einer sehr kleinen Anzahl von Patienten in einem bestens aufeinander abgestimmten System aus interventioneller Kardiologie im Krankenhaus und hochgradig standardisierter präklinischer Versorgung durchgeführt wurde. Die alleinige Etablierung einer ECMOTherapie, die nicht von einer derart koordinierten Versorgung flankiert wird, ließe vor diesem Hintergrund wohl nicht dieselben positiven Ergebnisse erwarten.

\section{Fazit für die Praxis}

- SGLT2(„sodium dependent glucose cotransporter $2^{\prime \prime}$ )-Inhibitoren stellen unabhängig vom Vorliegen eines Diabetes mellitus eine wichtige neue medikamentöse Therapieoption für HFrEF(Herzinsuffizienz mit eingeschränkter Ejektionsfraktion)-Patienten dar; für HFpEF (Herzinsuffizienz mit erhaltener Ejektionsfraktion) gibt es erste positive Signale.

- Sacubitril/Valsartan kommt auch als First-line-Therapie bei HFrEF infrage.

- Neue Studien berichten positive Effekte für sGC(lösliche Guanylatcyclase)-Stimulatoren und Myosinaktivatoren.

- Die Edge-to-edge-Mitralklappenreparatur stellt eine wichtige Option zur interventionellen Herzinsuffizienztherapie dar.

- Mit der Barorezeptoraktivierungstherapie und neuen perkutan implantierbaren Unterstützungssystemen eröffnen sich weitere interessante nichtpharmakologische Therapiekonzepte für die chronische und akute Herzinsuffizienz. 


\section{Korrespondenzadresse}

PD Dr. Christoph Birner
Klinik für Innere Medizin I,
Klinikum St. Marien Amberg
Mariahilfbergweg 7,
92224 Amberg, Deutschland
birner.christoph@klinikum-
amberg.de

\section{Einhaltung ethischer Richtlinien}

Interessenkonflikt. U. Laufs: in den letzten 5 Jahren Honorare für klinische Studien, Vorträge oder Beratung an U. Laufs oder Mitarbeiter, das Universitätsklinikum Leipzig, Homburg/Saar oder die Medizinische Fakultät Leipzig von folgenden Firmen: Amgen, AstraZeneca, Bayer, Boehringer, Novartis, Servier. N. Frey: Vortragshonorare von AstraZeneca und Böhringer-Ingelheim. C. Birner gibt an, dass kein Interessenkonflikt besteht.

Für diesen Beitrag wurden von den Autoren keine Studien an Menschen oder Tieren durchgeführt. Für die aufgeführten Studien gelten die jeweils dort angegebenen ethischen Richtlinien.

\section{Literatur}

1. Ambrosy AP, Braunwald E, Morrow DA, DeVore AD, McCague K, Meng X, Duffy Cl, Rocha R, VelazquezEJ (2020) Angiotensin receptor-Neprilysin inhibition based on history of heart failure and use of ReninAngiotensin system antagonists. J Am Coll Cardiol 76(9):1034-1048. https://doi.org/10.1016/j.jacc. 2020.06.073

2. Armstrong PW, Lam CSP, Anstrom KJ, Ezekowitz J, Hernandez AF, O'Connor CM, Pieske B, PonikowskiP, Shah SJ, Solomon SD, Voors AA, She L, Vlajnic V Carvalho F, Bamber L, Blaustein RO, Roessig L, ButlerJ(2020) Effect of Vericiguat vs placebo on quality of life in patients with heart failure and preserved ejection fraction. The VITALITY-HFpEF randomized clinical trial. JAMA 324(15):1512-1521. https://doi. org/10.1001/jama.2020.15922

3. Armstrong PW, Pieske B, Anstrom KJ, Ezekowitz J, Hernandez AF, Butler J, Lam CSP, Ponikowski $P$, Voors AA, Jia G, McNulty SE, Patel MJ, Roessig L, Koglin J, O'Connor CM (2020) Vericiguat in patients with heart failure and reduced ejection fraction. N Engl J Med 382(20):1883-1893. https://doi.org/ 10.1056/NEJMoa1915928

4. Baumgartner H, Falk V, Bax JJ, de Bonis M, Hamm C, Holm PJ, lung $B$, Lancellotti $P$, Lansac $E$, Rodriguez Muñoz D, Rosenhek R, Sjögren J, Tornos Mas $P$, Vahanian A, Walther T, Wendler O, Windecker S, Zamorano JL (2017) 2017 ESC/EACTS Guidelines for the management of valvular heart disease. Eur Heart J 38(36):2739-2791. https://doi.org/10. 1093/eurheartj/ehx391

5. Bhatt DL, Szarek M, Steg PG, Cannon CP, Leiter LA, McGuire DK, Lewis JB, Riddle MC, Voors AA, Metra M, Lund LH, Komajda M, Testani JM, Wilcox CS, Ponikowski P, Lopes RD, Verma S, Lapuerta P, Pitt B (2020) Sotagliflozin in patients with diabetes and recent worsening heart failure. N Engl J Med. https://doi.org/10.1056/ NEJMoa2030183
6. Chen T, Wu D, Chen H, Yan W, Yang D, Chen G, Ma K, Xu D, Yu H, Wang H, Wang T, Guo W, Chen J, Ding C, Zhang X, Huang J, Han M, Li S, Luo X, Zhao J, Ning Q (2020) Clinical characteristics of 113 deceased patients with Coronavirus disease 2019. Retrospective study. BMJ. https://doi.org/ 10.1136/bmj.m1091

7. Dörr M (2020) Hospitalizations due to heart failure: major differences between East and West Germany remaining even 30 years after reunification. Pressemitteilung derESC

8. Godino C, Munafò A, Scotti A et al (2020) MitraClip in secondary mitral regurgitation as a bridge to heart transplantation. 1-year outcomes from the International MitraBridge Registry. J Heart Lung Transplant 39(12):1353-1362. https://doi.org/10 1016/j.healun.2020.09.005

9. McMurray JJV, Packer M, Desai AS, Gong J, Lefkowitz MP, Rizkala AR, Rouleau JL, Shi VC, Solomon SD, Swedberg K, Zile MR (2014) Angiotensin-neprilysin inhibition versus enalapri in heart failure. N Engl J Med 371(11):993-1004. https://doi.org/10.1056/NEJMoa1409077

10. McMurray JJV, Solomon SD, Inzucchi SE et al (2019) Dapagliflozin in patients with heart failure and reduced ejection fraction. $\mathrm{N}$ Engl J Med 381(21):1995-2008. https://doi.org/10.1056/ NEJMoa1911303

11. Packer M, Anker SD, Butler J et al (2020) Cardiovascular and renal outcomes with Empagliflozin in heart failure. N Engl J Med 383(15):1413-1424. https://doi.org/10.1056/NEJMoa2022190

12. Pieske B PARALLAX: Sacubitril/Valsartan versus Individualized RAAS Blockade in Patients with HFpEF. vorgestellt bei der HOTLINE II-Session am 30. Aug. 2020 beim ESC Congress 2020-The Digital Experience

13. Ponikowski $P$, Voors AA, Anker SD, Bueno $H_{\text {, }}$ Cleland JGF, Coats AJS, Falk V, González-Juanatey JR, Harjola V-P, Jankowska EA, Jessup $M$, Linde C, Nihoyannopoulos P, Parissis JT, Pieske B Riley JP, Rosano GMC, Ruilope LM, Ruschitzka F, Rutten FH, van der Meer P (2016) 2016 ESC Guidelines for the diagnosis and treatment of acute and chronic heart failure. The Task Force for the diagnosis and treatment of acute and chronic heart failure of the European Society of Cardiology (ESC)Developed with the special contribution of the Heart Failure Association (HFA) of the ESC. Eur Heart J 37(27):2129-2200. https://doi.org/10. 1093/eurheartj/ehw128

14. Schrage B, Becher PM, Bernhardt A et al (2020) Left ventricular unloading is associated with lower mortality in patients with cardiogenic shock treated with venoarterial extracorporeal membrane oxygenation. Results from an international, multicenter cohort study. Circulation 142(22):2095-2106. https://doi.org/10.1161/ CIRCULATIONAHA.120.048792

15. Seferović PM, Fragasso G, Petrie M et al (2020) Heart Failure Association of the European Society of Cardiology update on sodium-glucose cotransporter 2 inhibitors in heart failure. Eur J Heart Fail 22(11):1984-1986. https://doi.org/10.1002/ ejhf.2026

16. Senni M, Wachter R, Witte KK, StraburzynskaMigaj E, Belohlavek J, Fonseca C, Mueller C, Lonn E, Chakrabarti A, Bao W, Noe A, Schwende H, Butylin D, Pascual-Figal D (2020) Initiation of sacubitril/valsartan shortly after hospitalisation foracutely decompensated heartfailure in patients with newly diagnosed (de novo) heart failure. A subgroup analysis of the TRANSITION study. Eur J Heart Fail 22(2):303-312. https://doi.org/10.1002/ ejhf.1670
17. Solomon SD, McMurray JJV, Anand IS et al (2019) Angiotensin-Neprilysin inhibition in heart failure with preserved ejection fraction. N Engl J Med381(17):1609-1620.https://doi.org/10.1056/ NEJMoa1908655

18. Stone GW, Lindenfeld J, Abraham WT, Kar S, Lim DS, Mishell JM, Whisenant B, Grayburn PA, Rinaldi M, Kapadia SR, Rajagopal V, Sarembock IJ, Brieke A, Marx SO, Cohen DJ, Weissman NJ, Mack MJ (2018) Transcatheter mitral-valve repair in patients with heart failure. N Engl J Med 379(24):2307-2318. https://doi.org/10.1056/NEJMoa1806640

19. Teerlink JR, Diaz R, Felker GM et al (2020) Cardiac myosin activation with Omecamtiv Mecarbil in systolic heart failure. N Engl J Med. https://doi.org/ 10.1056/NEJMoa2025797

20. Teerlink JR, Diaz R, Felker GM, McMurray JJV, Metra M, Solomon SD, Legg JC, Büchele G, Varin C, Kurtz CE, Malik Fl, Honarpour N (2020) Omecamtiv Mecarbil in chronic heart failure with reduced ejection fraction. Rationale and design of GALACTIC-HF. JACC Heart Fail 8(4):329-340. https://doi.org/10.1016/j.jchf.2019.12.001

21. Udelson JE, Lewis GD, Shah SJ, Zile MR, Redfield MM, Burnett J, Parker J, Seferovic JP, Wilson P, Mittleman RS, Profy AT, Konstam MA (2020) Effect of Praliciguat on peak rate of oxygen consumption in patients with heart failure with preserved ejection fraction. The CAPACITY HFpEF randomized clinical trial.JAMA 324(15):1522-1531. https://doi. org/10.1001/jama.2020.16641

22. Wachter R, Senni M, Belohlavek J, StraburzynskaMigaj E, Witte KK, Kobalava Z, Fonseca C, Goncalvesova E, Cavusoglu Y, Fernandez A, Chaaban S, Bøhmer E, Pouleur A-C, Mueller C, Tribouilloy C, Lonn E, Buraiki J, Gniot J, Mozheiko M, Lelonek M, Noè A, Schwende H, Bao W, Butylin D, Pascual-Figal D (2019) Initiation of sacubitril/ valsartan in haemodynamically stabilised heart failure patients in hospital or early after discharge. Primary results of the randomised TRANSITION study. Eur J Heart Fail 21(8):998-1007. https://doi. org/10.1002/ejhf.1498

23. Wachter R, Klebs S, Balas B, Kap E, Engelhard J, Schlienger R, Wirta BS, Fonseca AF (2020) Heart failure signs and symptoms, hospita referrals, and prescription patterns in patients receiving sacubitril/valsartan in primary care and cardiologist settings in Germany. ESC Heart Fail 7(5):2318-2330. https://doi.org/10.1002/ehf2. 12768

24. Yannopoulos D, Bartos J, Raveendran G, Walser E, Connett J, Murray TA, Collins G, Zhang L, Kalra R, Kosmopoulos M, John R, Shaffer A, Frascone RJ, Wesley K, Conterato M, Biros M, Tolar J, Aufderheide TP (2020) Advanced reperfusion strategies for patients with out-of-hospital cardiac arrest and refractory ventricular fibrillation (ARREST). A phase 2 , single centre, open-label, randomised controlled trial. Lancet 396(10265):1807-1816. https://doi.org/10.1016/S0140-6736(20)32338-2

25. Zile MR, Lindenfeld J, Weaver FA, Zannad F, Galle E, Rogers T, Abraham WT (2020) Baroreflex activation therapy in patients with heart failure with reduced ejection fraction. J Am Coll Cardiol 76(1):1-13. https://doi.org/10.1016/j.jacc.2020.05.015

26. Zinman B, Wanner C, Lachin JM, Fitchett D, Bluhmki E, Hantel S, Mattheus M, Devins T, Johansen OE, Woerle HJ, BroedI UC, Inzucchi SE (2015) Empagliflozin, Cardiovascular Outcomes, and Mortality in Type 2 Diabetes. N Engl J Med 373(22):2117-2128.https://doi.org/10.1056/ NEJMoa1504720 